\title{
Magnitude, Pattern and Factors Associated with Ocular Manifestations in Pre-eclampsia/Eclampsia at Mulago National Referral Hospital, Uganda
}

Beatrice M Bako1*, Anne Ampaire ${ }^{1}$, Annette Nakimuli $^{2}$, Charity Zalwango ${ }^{1}$, Joanita Nalunjonji ${ }^{4}, \mathrm{Moses}$ Kasadhakawo ${ }^{3}$

${ }^{1}$ Department of Ophthalmology, College of Health Sciences, Makerere University, Uganda

${ }^{2}$ Department of Ophthalmology, Department of Obstetrics and Gynecology, Makerere University, Uganda

${ }^{3}$ Mulago National Referral Hospital, Department of Ophthalmology, Uganda

${ }^{4}$ Makerere University Lung Institute, College of Health Sciences, Uganda

*Corresponding Author: Bako Monday Beatrice, Department of Ophthalmology, College of Health Sciences, Makerere University, Uganda; Email: bmbbeat@hotmail.com

Received Date: 14-03-2021; Accepted Date: 05-04-2021; Published Date: 13-04-2021

Copyright $^{\odot} 2021$ by Beatrice BM, et al. All rights reserved. This is an open access article distributed under the terms of the Creative Commons Attribution License, which permits unrestricted use, distribution, and reproduction in any medium, provided the original author and source are credited.

\begin{abstract}
Background: Preeclampsia is a condition where expectant mothers experience a sudden spike in blood pressures and in its severe form of eclampsia they tend to experience seizures. Over $25 \%$ of patients with severe pre-eclampsia and $50 \%$ of patients with eclampsia have visual symptoms of clinical significance, with 1-3\% remaining with irreversible blindness due to involvement of the visual cortex. The ocular changes in preeclampsia usually occur from $20+$ weeks of gestation and progress gradually. Hence, early screening of pre-eclampsia mothers can help in timely diagnosis and intervention. With limited data on the ocular conditions of mothers with preeclampsia, this study sought to determine the magnitude, pattern and factors associated with ocular manifestations of preeclampsia/eclampsia mothers at Mulago National Referral Hospital.
\end{abstract}

Methods: This was a descriptive cross-sectional study with 450 consenting pre-eclampsia mothers at Mulago National Referral Hospital from September to December. Ethical Approval was obtained, and pre-tested questionnaires were used to record relevant history and ocular examination findings. Data was cleaned and entered into a computer in EPI-DATA (epidemiology data) and analyzed with STATA 14.0. 
Results: The prevalence of ocular manifestations was $16.5 \%$ CI (13.3-20.2\%). The commonest patterns of ocular manifestations were optic disc edema 19 (26.7\%), retinal hemorrhages $17(22.9 \%)$ and cotton wool spots $16(21.6 \%)$. The factors associated with ocular manifestation of preeclampsia/eclampsia were education ( $\mathrm{p}$-value $=0.042$ ), sub-urban residence ( $\mathrm{p}$ value $=0.045$ ), severe preeclampsia and eclampsia ( $\mathrm{p}$-values $=0.008$ and 0.00 ), abnormal liver function test ( $\mathrm{p}$-value $=0.002$ ) and abnormal visual acuity ( $\mathrm{p}$-value $=0.000$ ).

Conclusion: The prevalence of ocular manifestations of pre-eclampsia and eclampsia among women in Mulago National Referral hospital is high and warrants active screening programmes.

\section{Keywords}

Preeclampsia; Eclampsia; Hypertension; Hemorrhage

\section{Introduction}

Preeclampsia is the new onset of hypertension with either proteinuria, end-organ dysfunction or both after 20 weeks of gestation in a previously normotensive woman while eclampsia is development of grand mal seizures in a woman with pre-eclampsia, in the absence of other neurological conditions that could account for the seizures.

Globally eclampsia accounts for $12 \%$ maternal mortality mostly in the developing countries where the incidence of eclampsia is high and quality of care of pre-eclamptic women is low [1].

The exact prevalence of preeclampsia in Sub Saharan Africa (SSA) is unknown because detailed clinical records of all births are lacking. Using information from the Duke University Birth Database, African American women had higher rates of preeclampsia (10.2\%) than the European $(8 \%)$ or Hispanic women $(6.2 \%)$.

The symptoms of pre-eclampsia include headache, fits and photophobia. These symptoms have been found to be associated with ocular changes including blurring of vision and ischemia in the retina. These ocular changes have been shown to predict poor outcomes including death, blindness among others in case treatment is delayed [2].

Pre-existing ocular and systemic diseases such as chronic systemic hypertension and diabetic retinopathy are risk factors for development of ocular complications in pre-eclampsia and eclampsia.

Ocular changes can be screened among pre-eclampsia mothers however in Mulago National Referral Hospital (MNRH), pre-eclampsia/eclampsia mothers are hardly screened for ocular changes.

Beatrice BM | Volume 2; Issue 1 (2021) | JOAR-2(1)-011 | Research Article 
Therefore, this study sought to determine the magnitude, patterns and factors associated with ocular changes of pre-eclampsia/eclampsia in MNRH.

\section{Methods}

This was a hospital based descriptive cross-sectional study conducted between September and December at Mulago National Referral Hospital in Kampala, Uganda.

Data was collected from the pre-eclampsia wards (mild pre-eclampsia, severe pre-eclampsia and high dependence unit) which on average admit 36 preeclampsia patients per month.

All pregnant women diagnosed with preeclampsia/eclampsia of any parity admitted in the preeclampsia wards in the study period were consecutively consented for participation in the study.

Patient with history of hypertension, glaucoma, sickle cell disease, corneal scars, severe posterior synechea, dense cataracts, and vitreous opacities were excluded from the study.

Data was collected using pretested questionnaires. A short medical, full obstetric and ocular history was obtained from the patient. General examination and bilateral standard ocular examination was done. Visual acuity was assessed using a Snellen's chart. Best corrected visual acuity was assessed using a pin hole. The intraocular pressure was measured using an icare tonometer. Fundus photos were taken using an I-phone with an installed fundus camera, software called volk iNview. These were reviewed by the ophthalmologist to confirm diagnosis.

Investigations done included random blood sugar, liver function and renal function tests.

All data were entered into a computer using EPI-DATA. Analysis was done with STATA version 14.0. Logistic regression was used to determine the factors associated with ocular abnormalities among women with preeclampsia. All factors with p-value $<0.05$ were considered significant.

Approval to conduct the study was obtained the School of Medicine Ethics and Research Committee (SOMREC) of Makerere University.

\section{Results}

A total of 450 pre-eclamptic/eclamptic mothers participated in the study. The age range was 18-42 years with a median of 27 years. The prevalence of ocular Manifestations was $16.5 \%$ CI (13.3-20.2\%) (Table 1). 


\begin{tabular}{|c|c|c|}
\hline Ocular Manifestation & Frequency & Percentage (\%) \\
\hline Optic disc edema & 19 & 26.7 \\
\hline Retinal hemorrhages & 17 & 21.6 \\
\hline Cotton wool spots & 16 & 5.0 \\
\hline Optic disc pallor & 4 & 4 \\
\hline Aneurysms & 3 & 4.0 \\
\hline Arteriolar narrowing & 3 & 16.1 \\
\hline Others & 12 & \\
\hline
\end{tabular}

Table 1: The distribution of ocular manifestations among 74 mothers with Pre-eclampsia /eclampsia.

There were 74 participants diagnosed with ocular manifestations of pre-eclampsia/eclampsia. The total number of participants with hypertensive retinopathy was $54(78.3 \%)$.

The commonest ocular manifestations of pre-eclampsia/eclampsia were optic disc edema (25.68\%), retinal hemorrhages $(22.97 \%)$, cotton wool spots $(21.62 \%)$. arteriolar narrowing $(4.05 \%)$, aneurysms (4\%) and optic disc pallor (5\%).

$59 \%$ ( 44) of the mothers with ocular manifestations needed a review by an ophthalmologist while $22.9 \%$ would benefit from a close follow up (Table 2).

\begin{tabular}{|c|c|c|c|}
\hline Variable & Odds Ratio & 95\% CI & P-value \\
\hline Education & 1.00 & & \\
None & 0.11 & $0.01-0.92$ & $0.042^{*}$ \\
Educated & 1.00 & & \\
\hline Residence & 0.31 & $0.10-1.00$ & $0.045^{*}$ \\
Rural & 0.53 & $0.21-1.37$ & 0.193 \\
Sub-urban & 1.00 & & \\
Urban & & \\
\hline Severity of Pre-eclampsia & & & \\
\hline
\end{tabular}

Beatrice BM | Volume 2; Issue 1 (2021) | JOAR-2(1)-011 | Research Article

Citation: Beatrice BM, et al. Magnitude, Pattern and Factors Associated with Ocular Manifestations in

Pre-eclampsia /Eclampsia at Mulago National Referral Hospital, Uganda. J Ophthalmol Adv Res.

2021;2(1):1-7.

DOI: http://dx.doi.org/10.46889/JOAR.2021.2106 


\begin{tabular}{|c|c|c|c|}
\hline $\begin{array}{c}\text { Eclampsia } \\
\text { Severe }\end{array}$ & $\begin{array}{c}34.53 \\
6.38\end{array}$ & $\begin{array}{c}7.54-158.18 \\
1.62-25.11\end{array}$ & $0.000^{*}$ \\
\hline $\begin{array}{c}\text { Liver Function tests } \\
\text { Normal }\end{array}$ & 1.00 & & $0.002^{*}$ \\
Abnormal & 4.13 & $1.71-10.00$ & \\
\hline Visual Acuity & 1.00 & & $0.000^{*}$ \\
Normal & 22.61 & $9.66-52.89$ & \\
\hline Abnormal & & & \\
\hline
\end{tabular}

Table 2: Factors associated with ocular manifestations in patients with preeclampsia/eclampsia.

Interaction was assessed among significant predictors using the likelihood ratio test. A p-value $<0.05$ confirmed the presence of interaction. There was no interaction.

There was a statistically significant relationship between the ocular manifestations of preeclampsia and education, severity of pre-eclampsia, liver function test, visual acuity and semiurban residence.

\section{Discussion}

Among the 450 mothers with pre-eclampsia/eclampsia, the prevalence of ocular manifestations was $16.5 \%$. The prevalence obtained in our study was lower compared to the $46 \%$ obtained in a study done in India by Reddy Sagili among 78 patients with pregnancy induced hypertension [3].

The prevalence obtained in our study was still lower than the $42.7 \%$ obtained in a study by Varija $\mathrm{T}$, et al., in a cross-sectional study involving 423 mothers with pregnancy induced hypertension. The study was conducted in a hospital setting in Karnataka India. The difference in the results obtained may be due to the differences in the populations studied. The study in India involved participants who were older with an age range of 21-45 years compared to 1842 years observed in our study. This may indicate that the mothers in Uganda present with less severe forms of pre-eclampsia at an earlier age compared to those in India. In addition only $30 \%$ of the population of mothers in our study were prime-gravidae compared to $66.7 \%$ of those studied by Varija, et al. This further points to a difference in the two populations [4]. 


\section{Patterns of Ocular Manifestations}

Most of ocular manifestations among patients with pre-eclampsia/eclampsia observed in our study were $25 \%$ optic disc edema, $22.2 \%$ hemorrhage and $21 \%$ cotton wool spots among others. The findings in our study are similar to those of Sharma, et al., where hemorrhage, optic edema and cotton wool spots were the common presentation of ocular abnormalities of preeclampsia/eclampsia [5]. In addition, findings from our study compare favourably with the results of an article about pregnancy and ocular manifestations done by Gordin, et al., in 2013 which emphasizes pre-eclampsia/ eclampsia as one of the causes of various ocular manifestations [6,7].

Our study however found no retinal detachment among all the participants investigated which is one of the ocular presentations of pre-eclampsia. This may be explained by the fact that most of the study participants had pre-eclampsia yet retinal detachment is common in eclampsia complicated by the HELLP syndrome [8].

Individuals with abnormal visual acuity were up to twenty-two times more likely to have ocular manifestations compared to those with normal visual acuity. Our finding of abnormal vision is in agreement with findings in a systematic review done by Roos to determine the visual disturbance of pre-eclampsia which found abnormal spontaneous inability to see in a 24 year old mother at 37 weeks [9].

A case study done by Tranos to determine visual acuity and other ocular manifestation in a prime gravida found normal visual acuity despite retinal detachment. These results show that some patients with pre-eclampsia also have normal visual acuity [10]. The results in our study had few mothers with visual impairment $20.7 \%$ compared with those of a study done in Muhimbili hospital, which found up to $35.4 \%$ visual impairment among mothers with preeclampsia/ eclampsia [11].

Eighty eight percent of the mothers had normal liver function test while 28(48.3\%) had abnormal liver function test. Mothers with abnormal liver function were found to be four times ( $\mathrm{p}$-value $=0.002$ ) more likely to develop ocular manifestations of pre-eclampsia compared to those with normal liver function tests. These findings were similar to findings by Gupta, et al., from a retrospective cohort study among 40 patients with pre-eclampsia where they found uric acid p-value of 0.022 in the pre-eclampsia group [12].

We observed an increase in the odds of ocular manifestations for a unit increase in the grade of preeclampsia/eclampsia of up to 6 times as the grade of pre-eclampsia/eclampsia increased from none to severe pre-eclampsia. These findings were comparable with findings from studies done among individuals with pregnancy induced hypertension and pre-eclampsia/eclampsia which found an increase in ocular manifestations of pre-eclampsia as the severity of preeclampsia/eclampsia increased [12].

Our findings were also in agreement with findings from studies done by Ling and James, among individuals with pre-eclampsia/eclampsia which found increased prevalence of

Beatrice BM | Volume 2; Issue 1 (2021) | JOAR-2(1)-011 | Research Article

Citation: Beatrice BM, et al. Magnitude, Pattern and Factors Associated with Ocular Manifestations in

Pre-eclampsia /Eclampsia at Mulago National Referral Hospital, Uganda. J Ophthalmol Adv Res.

2021;2(1):1-7.

DOI: http://dx.doi.org/10.46889/JOAR.2021.2106 
intraocular manifestations e.g. Roth's spots. The odds of Roth's spots increased with increase in severity of pre-eclampsia/eclampsia [13].

A Study done by Kaliaperumal to obtain associations between pre-eclampsia/eclampsia and retinopathy have found an association between severity of pre-eclampsia and retinopathy. These findings are in tandem with findings from our study and indicate that retinopathy is associated with severity of pre-eclampsia [14].

\section{Conclusion}

The magnitude of ocular manifestations of pre-eclampsia and eclampsia among women in Mulago national Referral Hospital is high that warrants routine screening.

\section{References}

1. Mushumbusi EF, Mafwiri MM, Sanyiwa AJ, Kisimbi SJ, Mosenene S, Padhan DH, et al. Ocular manifestations among patients with pre-eclampsia and eclampsia at Muhimbili National Hospital; magnitude and presentations. The J Ophthalmol Eastern, Central and Southern Africa. 2016;20(1).

2. Kiondo P, Wamuyu-Maina G, Bimenya GS, Tumwesigye NM, Wandabwa J, Okong P. Risk factors for preeclampsia in Mulago Hospital, Kampala, Uganda. Tropical Medicine and International Health. 2012;17(4):480-7.

3. Reddy SC. Fundus changes in pregnancy induced hypertension. Int J Ophthalmol. 2012;5(6):694.

4. Varija T, Vanaja D, Raghavenda B. A study of prevalence and association of fundus changes in pregnancy induced hypertension. Int J of Reproduction, Contraception, Obstetrics and Gynecology. 2017;5(5):1375-9.

5. Sharma AK, Bhatt S. Reversible blindness in severe preeclampsia and eclampsia. 2004.

6. Samra KA. The eye and visual system in pregnancy, what to expect? An in-depth review. Oman J Ophthalmol. 2013;6(2):87.

7. Gordin D, Kaaja R, Forsblom C, Hiilesmaa V, Teramo K, Groop P-H. Pre-eclampsia and pregnancy-induced hypertension are associated with severe diabetic retinopathy in type 1 diabetes later in life. Acta Diabetologica. 2013;50(5):781-7.

8. Mol BW, Roberts CT, Thangaratinam S, Magee LA, De Groot CJ, Hofmeyr GJ. Pre-eclampsia. The Lancet. 2016;387(10022):999-1011.

9. Roos NM, Wiegman MJ, Jansonius NM, Zeeman GG. Visual disturbances in (pre) eclampsia. Obstetrical and Gynecological Survey. 2012;67(4):242-50.

10. Tranos P, Wickremasinghe S, Hundal K, Foster P, Jagger J. Bilateral serous retinal detachment as a complication of HELLP syndrome. Eye. 2002;16(4):491.

11. Mushumbusi F. Ocular Manifestations among patients with pre-eclampsia and eclampsia at Muhibili National Hospital; Magnitude and presentations. J Ophthalmol Eastern Central and Southern Africa. 2016;20(1):2-7.

12. Gupta A, Kaliaperumal S, Setia S, Suchi ST, Rao VA. Retinopathy in preeclampsia: association with birth weight and uric acid level. Retina. 2008;28(8):1104-10.

13. Ling R, James B. White-centred retinal haemorrhages (Roth spots). Postgraduate Med J. 1998;74(876):5812.

14. Kaliaperumal S, Setia S, Gupta A, Rao V. Fetal birthweight and diastolic blood pressure: association with retinopathy in severe preeclampsia. European J Ophthalmol. 2008;18(5):809. 